\section{P4.97 UPTAKE OF, AND BARRIERS AND FACILITATORS TO, HIV TESTING IN AUSTRALIAN- AND VIETNAMESE-BORN ADULTS IN QUEENSLAND, AUSTRALIA}

${ }^{1}$ Sarah Blondell, ${ }^{2}$ Mark Griffin, ' Jo Durham. ${ }^{1}$ The University of Queensland, Brisbane, Australia; ${ }^{2}$ Adasis, Brisbane, Australia

\subsection{6/sextrans-2017-053264.592}

Introduction In Australia, people born in South-East Asia have the highest proportion of late human immunodeficiency virus (HIV) diagnosis. Late diagnosis has important implications for prevention, treatment and management of HIV. Limited evidence is available, however, on the testing behaviours of the South-East Asian migrant population and general Australian population.

Methods The study used quantitative and qualitative methods to assess uptake of, and barriers and facilitators to, HIV testing in Vietnamese- and Australian-born adults (18-49 years) in Queensland, Australia. A 66- item self-administered survey was given to participants (Vietnamese: $n=188$ and Australian: $\mathrm{n}=195$ ) at selected sites in Brisbane using convenience sampling. The survey included demographic, HIV knowledge, HIV stigma, HIV risk, HIV testing and healthcare access items. Materials were available in Vietnamese and English. Qualitative interviews $(n=8)$ were done with Vietnamese-born survey participants to further explore survey findings.

Results No statistically significant differences were seen between the two groups in terms of ever tested, ever recommended a test, self-testing or same day testing. Vietnameseborn people were significantly more likely to have recently tested and to accept provider-initiated testing and counselling (PITC). Interview data also suggested a preference for PITC in the Vietnamese-born community, due to their trust in doctors. They also indicated potential high-risk sexual practices in Vietnamese-born men.

Conclusion The Australian- and Vietnamese-born populations had similar HIV testing practices. Preference for PITC, however, was greater in the Vietnamese-born population. PITC may help reduce late HIV diagnosis by overcoming a number of barriers associated with client-initiated testing, e.g. poor risk perception and knowledge. Further research on the preference for PITC in this population and with healthcare providers who would be offering PITC is needed. Additionally, research is needed into the risk of HIV exposure when travelling back to Vietnam.

\section{P4.98 SPIRO, A PATIENT-MANAGED MOBILE APPLICATION TO IMPROVE SYPHILIS TREATMENT FOLLOW-UP AMONG MEN WHO HAVE SEX WITH MEN}

${ }^{1}$ Sebastiaan Hulstein, ${ }^{1}$ Menne Bartelsman, ${ }^{1}$ Freke Zuure, ${ }^{1}$ Udi Davidovich, ${ }^{1}$ Amy Matser, ${ }^{1}$ Arjan Hogewoning, ${ }^{2}$ Henry DE Vries. ${ }^{1}$ Public Health Service of Amsterdam (GGD Amsterdam), Amsterdam, The Netherlands; ${ }^{2}$ Academic Medical Centre, Department of Dermatology, Amsterdam, The Netherlands

\subsection{6/sextrans-2017-053264.593}

Introduction Syphilis incidence is rising in developed countries worldwide, and men who have sex with men (MSM) are disproportionally affected. Follow-up after treatment of syphilis is important as late complications (e.g. neurosyphilis), therapy failure and re-infections can occur, especially in HIV coinfected patients. Failure to complete follow-up occurs frequently. Moreover, medical history at follow-up is often incomplete, as follow-up occurs often at various health care providers. Self-management of the relevant medical data might improve patient engagement, and ensure the availability of medical data required during follow-up visits.

Methods We developed a patient-managed mobile phone application - Spiro - that can be used by patients treated for syphilis, to record relevant data for follow-up like medical history, physical findings, treponomal (e.g. TTPA) and non-treponomal (e.g. VDRL) serum antibody results, conclusions and treatment. Spiro automatically summarises the data relevant for syphilis follow-up including a graphical depiction of the nontreponomal serum antibody titer over time. Follow-up data are stored locally on the mobile device. Optionally, encrypted online storage is available. Lay information on risk factors for, and symptoms, diagnosis and treatment of syphilis infections is available via the app.

Results After launching Spiro on World AIDS day 2016, we intend to measure its acceptability and usability among patients who use the app and their care providers. In addition, we plan to evaluate retention to follow-up, the availability of required data for syphilis treatment follow-up, healthliteracy and self-reported level of disease control among MSM newly diagnosed with syphilis in a multi-centre RCT, comparing standard care plus the use of Spiro by patients to standard care alone.

Conclusion If feasible and effective, Spiro, a newly developed medical mobile phone application, can improve the engagement in, and retention to syphilis treatment follow-up among MSM.

\section{P4.99 HIV STATUS DOES IT MAKE A DIFFERENCE? SEXUAL BEHAVIOUROF HIV POSITIVE MEN ATTENDING A COMPREHENSIVE TREATMENT CENTRE IN LAGOS, NIGERIA}

${ }^{1}$ Sekoni Adekemi, ${ }^{1}$ Uthman Oluwaseun, ${ }^{2}$ Somefun Esther. 'University of Lagos, Lagos, Nigeria; ${ }^{2}$ Lagos University Teaching Hospital, Lagos, Nigeria

\subsection{6/sextrans-2017-053264.594}

Introduction In many sub-Saharan African countries, most new cases of Human Immunodeficiency Virus (HIV) infection occur in HIV sero-discordant couples. This study assessed the sexual behaviour of HIV positive men accessing comprehensive services at Nigerian Institute of Medical Research Lagos.

Methods A cross sectional study was carried out among men accessing ARV at a PEPFAR Clinic. Ethical approval was obtained. Respondents were recruited consecutively on clinic days among the attendees until calculated sample size of 384 was attained. Data was analysed using IBM SPSS version 20. Association was explored using $\mathrm{p}<0.05$.

Results The mean age was 43.06 years. $74.8 \%$ were married at diagnosis, $58.1 \%$ tested with their partner, $78.5 \%$ knows the HIV status of their partners and $79.2 \%$ had been on ARV for more than a year. With respect to sexual behaviour, $38.9 \%$ and $34.3 \%$ engage in transactional and intergenerational sex respectively. $21.1 \%$ and $8.3 \%$ were into concurrent multiple sexual partnerships in the twelve months and three months preceding the survey. $7.2 \%$ had sex with casual partners or sex workers, $45.4 \%$ did not use condom consistently, among these men, $18.1 \%$ did not use condom at last sex and the last sexual partner for $7.9 \%$ was a sex worker/casual friend. Unmarried respondents were more likely to have 